\title{
THE FORWARD RATES FOR MULTIFACTOR MODEL OF TERM STRUCTURE “WITH SQUARE ROOT”
}

\author{
Medvedev Gennady \\ Belarusian State University \\ 4 F. Skorina ave. Minsk, 220050, Belarus \\ Tel:+0375172095448 \\ MedvedevGA@bsu.by
}

\begin{abstract}
The multifactor model "with square root" is discussed in details. For such model, the representation of state variable process in the integral form is derived and its covariance matrix is found. The special attention to the problem connected with the tendency for the term structure of long-term forward rates to slope downwards is given.

For multifactor models with square root the following results are derived: representations of the forward rate curve through the volatility of the state variable process and through the volatility of zero coupon yield process are obtained; the expectations, variances and covariances for the forward rates and the yield process volatility are calculated; the expectation and the variance for the derivative of forward rate are found; the Brown - Schaefer approximation for the spread of forward rate is examined.

As examples two three-factor models (BDFS and Chen models) are examined. On the basis of the estimates of parameters of these models received by empirical way the numerical analysis including calculation of covariance matrixes of process of state variables, calculation of an expectation of the local variance of yield process and calculation of the variance of zero coupon yield have been fulfilled.
\end{abstract}

Keywords: multifactor model of term structure, forward rate curve, volatility of zero coupon yield, affine model, square root model CIR, term structure of long-term forward rates.

\section{Introduction}

One of classical problems of financial economics is the analysis of behavior of the yield on default free bonds depending on their maturities. At the certain assumptions it is possible to use mathematical model of available yield curve to extrapolate it to obtain the future values of yield rates. The forward rates can be obtained on the basis of knowledge of term structure of discount bonds (see details in Hull (1993)). Properties of forward curves for one factor models of term structure in details are considered in many papers. Here we shall refer only to Medvedev (2003), Medvedev (2004), as present paper is direct extension of these results on multifactor model "with square root".

The area of term structure for long-term forward rates was subjected to detailed research by Brown and Schaefer (2000), which have discovered that the new information about the yield term structure can be received from the analysis of the long-term end of the forward rate curve. Using a two factor Gaussian model they had shown that the long term forward rate curve is downward sloping whenever the volatility of the long term zero coupon yield is sufficiently high. They had verified this for real data on US Treasury STRIPs.

In the present paper the analysis of the forward rate curve is made for class of affine multifactor models of the term structure that are generated by the short term (riskless) interest rate model "with square root". 


\section{Analysis of Multifactor Model}

Let us assume that the dynamics model of riskfree rates $r(t)$ belongs to a class of models "with square roots" and is characterized by $n$ state variables which form a vector $Z=\left(z_{1}, \ldots\right.$, $\left.z_{n}\right)^{\mathrm{T}}$, i.e. $r(t)=r(Z(t))$. For affine model it is necessary that $r(Z)$ was affine function of state parameters, i.e. $r(Z)=\alpha+\phi^{\mathrm{T}} Z, \phi^{\mathrm{T}}=\left(\phi_{1}, \phi_{2}, \ldots, \phi_{n}\right)$. For such $n$-factor model with constant coefficients the state variables follow the stochastic differential equation

$$
d Z=K(\theta-Z) d t+\sigma \sqrt{<\delta+\Gamma Z>} d W(t),
$$

where $K$ is $(n \times n)$-matrix of the mean reversion coefficients; $\theta$ is $n$-vector of stationary expectations of state variables $Z ; d W$ is $q$-vector of increments of standard Brownian motions; $[\sigma \sqrt{<\delta+\Gamma Z>}]$ is $(n \times q)$-matrix of volatilities; $\sigma$ is $(n \times q)$-matrix; $\delta$ is $q$-vector, $\Gamma$ is $(q \times n)$ matrix of coefficients of state variable influences on volatility; symbol $<\delta>$ designs a diagonal matrix, on main diagonal which there are components of vector $\delta$. This means that

$$
<\delta+\Gamma Z>_{i j}=\left\{\begin{array}{cl}
\delta_{i}+\sum_{k=1}^{n} \Gamma_{i k} Z_{k}, & \text { if } i=j \\
0 & \text { if } i \neq j .
\end{array}\right.
$$

It is assumed that for equation (1) the existence and uniquely conditions are held (see Duffie and Kan (1996)). By classification Dai and Singleton (2000) this model belongs to class $A_{m}(n), m$ is rank of the matrix $\Gamma$, and for the specification in the maximal variant the model demands to set $(n \times n)$-matrix, $(n \times q)$-matrix, $(q \times n)$-matrix, $q$-vector, and $n$-vector, i.e. $n(1+2 q+n)+q$ parameters.

If the state of process $Z$ at some moment of time $s<t$ is known then such model allows to express a vector of state variables $Z(t)$ in the integral form as process

$$
Z(t)=U(t-s) Z(s)+(I-U(t-s)) \theta+\int_{s}^{t} U(t-u) \sigma \sqrt{<\delta+\Gamma Z(u)\rangle} d W(u),
$$

where $U(t)$ is the fundamental $(n \times n)$-matrix of solutions for the ordinary differential equation $U^{\prime}(t)=-K U(t), U(0)=I, I$ is identity $(n \times n)$-matrix. The stationary regime of such process exists if all eigenvalues of matrix $K$ are negative or have the negative real parts (in this case at $t \rightarrow \infty$ matrix $U(t) \rightarrow 0)$. For a stationary regime $(s \rightarrow-\infty)$ the expression for process $Z(t)$ becomes more simple

$$
Z(t)=\theta+\int_{0}^{\infty} U(u) \sigma \sqrt{<\delta+\Gamma Z(t-u)>} d W(t-u)
$$

whence follows that the unconditional expectation and the unconditional covariance matrix of process $Z(t)$ are calculated by formulae

$$
E[Z(t)]=\theta, \quad \operatorname{Cov}[Z(t)]=\int_{0}^{\infty} U(u) \sigma<\delta+\Gamma \theta>\sigma^{\mathrm{T}}[U(u)]^{\mathrm{T}} d u,
$$

If the eigenvalues of matrix $K$ are designed as $\beta_{i}<0,1 \leq i \leq n$, and the diagonal matrix with elements $\exp \left(\beta_{i} t\right)$ on the main diagonal is designed as $e^{\beta t}$ then the fundamental matrix of solutions $U(t)$ can be presented in the form $U(t)=M e^{\beta t} M^{-1}$ where $M$ is a matrix the columns of that form the eigenvectors of the matrix $K$. Note that if matrix $K$ is diagonal with elements $k_{i}>0$ on the main diagonal then $M=I, \beta_{i}=-k_{i}$, and $U(t)$ is equal to $e^{-k t}$.

The multifactor model of state variables (1) generates an affine model of term structure which can be written according to Duffie and Kan (1996) as 


$$
P(Z, t, \tau)=\exp \left[A(\tau)-Z^{\mathrm{T}} B(\tau)\right]
$$

where $Z=Z(t)$ and function $A(\tau)$ and vector $B(\tau)$ can be determined from the following differential equation for price $P(Z, t, \tau)$ :

$$
\frac{\partial P}{\partial t}+\frac{\partial P}{\partial Z} K(\theta-Z)+\frac{1}{2} \operatorname{tr}\left(\frac{\partial^{2} P}{\partial Z^{2}} \sigma<\delta+\Gamma Z>\sigma^{\mathrm{T}}\right)-r(Z)=\frac{\partial P}{\partial Z} \sigma<\delta+\Gamma Z>\lambda,
$$

where $\frac{\partial P}{\partial Z}=-B(\tau)^{\mathrm{T}}, \frac{\partial^{2} P}{\partial Z^{2}}=B(\tau) B(\tau)^{\mathrm{T}}$ are $n$-vector row and $(n \times n)$-matrix of derivatives of the price with respect to the state variables respectively, $r(Z)=\alpha+\phi^{\mathrm{T}} Z$ is the riskfree interest rate at the moment of time $t, \lambda-q$-vector of risk premium parameters at the assumption that the risk premium are defined by a relation $\lambda(Z)=\sqrt{\langle\delta+\Gamma Z>} \lambda$, and $\operatorname{tr}(A)$ is a trace of ma$\operatorname{trix} A$.

Under these conditions the functions $A(\tau)$ and $B(\tau)$ satisfy the ordinary differential equations

$$
\begin{aligned}
& A^{\prime}(\tau)=-\alpha-B(\tau)^{\mathrm{T}}[K \theta-\sigma<\delta>\lambda]+B(\tau)^{\mathrm{T}} \sigma<\delta>\sigma^{\mathrm{T}} B(\tau) / 2, A(0)=0, \\
& B^{\prime}(\tau)=\phi-\left[K^{\mathrm{T}}+\Gamma^{\mathrm{T}}<\lambda>\sigma^{\mathrm{T}}\right] B(\tau)-\Gamma^{\mathrm{T}}<B(\tau)^{\mathrm{T}} \sigma>\sigma^{\mathrm{T}} B(\tau) / 2, \quad B(0)=0 .
\end{aligned}
$$

Special interest is represented with functions $B(\tau)$ because through them the forward rates are expressed. However the solution of the vector equation (5) (vector Riccati equation) with respect to function $B(\tau)$ is not expressed in a closed form and the functions $B(\tau)$ can be determined only numerically.

\section{Forward Rate in Multifactor Model}

The forward rate curve $f(\tau \mid Z) \equiv-\partial \ln P(Z, \tau) / \partial \tau$ take the form

$$
\begin{gathered}
f(\tau \mid Z)=r(\theta)-B(\tau)^{\mathrm{T}} \sigma<\delta+\Gamma \theta>\lambda-\left[B(\tau)^{\mathrm{T}} \sigma<\delta+\Gamma \theta>\sigma^{\mathrm{T}} B(\tau)\right] / 2+ \\
+\left[\phi^{\mathrm{T}}-B(\tau)^{\mathrm{T}} K-B(\tau)^{\mathrm{T}} \sigma<\lambda>\Gamma-B(\tau)^{\mathrm{T}} \sigma<\sigma^{\mathrm{T}} B(\tau)>\Gamma / 2\right](Z-\theta),
\end{gathered}
$$

and the spread of forward rates $f\left(\tau_{2} \mid Z\right)-f\left(\tau_{1} \mid Z\right)$ for $\tau_{2}=\tau+\omega, \tau_{1}=\tau-\omega$ :

$$
\begin{gathered}
\Delta f(\tau, \delta) \equiv f\left(\tau_{2} \mid Z\right)-f\left(\tau_{1} \mid Z\right)= \\
=-\Delta B(\tau, \omega)^{\mathrm{T}}\left[\sigma<\delta+\Gamma \theta>\lambda+\sigma<\delta+\Gamma \theta>\sigma^{\mathrm{T}} \nabla B(\tau, \omega) / 2\right]- \\
-\Delta B(\tau, \omega)^{\mathrm{T}}\left[K+\sigma<\lambda>\Gamma+\sigma<\sigma^{\mathrm{T}} \nabla B(\tau, \omega)>\Gamma / 2\right](Z-\theta),
\end{gathered}
$$

where are used designations $\Delta B(\tau, \omega) \equiv B\left(\tau_{2}\right)-B\left(\tau_{1}\right), \nabla B(\tau, \omega) \equiv B\left(\tau_{2}\right)+B\left(\tau_{1}\right)$.

As the multifactor model is derived by the vector of the Brownian motions the yield process volatility is determined by the vector-row $\sigma_{y}(\tau)=B(\tau)^{\mathrm{T}} \sigma \sqrt{<\delta+\Gamma Z>} / \tau$ that is stochastic. For the forward rate it is possible to write the formula through the yield process volatility as follows:

$$
f(\tau \mid Z)=r(Z)+B(\tau)^{\mathrm{T}} K(\theta-Z)-\tau \sigma_{y}(\tau) \sqrt{<\delta+\Gamma Z>\lambda}-\tau^{2} \sigma_{y}(\tau) \sigma_{y}(\tau)^{\mathrm{T}} / 2 .
$$

The multifactor model of the riskfree interest rate process reflects the real dynamics more precisely however it demands to set the greater number of parameters and the explicit expression for the forward rate, volatility of yield process and unconditional variance of yield to maturity have rather bulky form. Therefore in order to obtain the visible results we will accept some simplifying assumptions.

Note first that as the state variables $Z$ it is necessary to choose only such variables, which influence on level of the riskfree interest rate. That is the vector $\phi$ should have only 
nonzero components. In this case without breaking a generality it is possible to represent the riskfree interest rate more simply by equivalent state variable: $r(Z)=\alpha+\mathbf{1}^{\mathrm{T}} \widetilde{Z}$, where $\mathbf{1}$ is a vector formed by units. Indeed let $\widetilde{Z} \equiv \Phi Z$, where $\Phi \equiv\langle\phi\rangle$ is a diagonal matrix the components of main diagonal of which are components of vector $\phi$. Then for the vector of state variables $\widetilde{Z}$ the equation of model (1) is obtained as

$$
d \widetilde{Z}=\widetilde{K}(\widetilde{\theta}-\widetilde{Z}) d t+\widetilde{\sigma} \sqrt{<\delta+\widetilde{\Gamma} \widetilde{Z}>} d W(t),
$$

where $\widetilde{K} \equiv \Phi K \Phi^{-1}, \widetilde{\theta} \equiv \Phi \theta, \widetilde{\sigma} \equiv \Phi \sigma$, and $W(t)$ is the same process as in model (1). The state variable $\widetilde{Z}$ have also the useful property that the eigenvalues of a matrix $\widetilde{K}$ are the same, as for $K$, and if a matrix $K$ is diagonal then $\widetilde{K}=K$. Other advantages of transition to the state variables $\widetilde{Z}$ we note later.

Consider a special case when the matrix $K$ is diagonal. In this case as it has above been told the elements of main diagonal are eigenvalues and the fundamental matrix of decisions $U(t)=e^{-k t}$ is too diagonal. Let a matrix $\sigma \sqrt{<\delta+\Gamma \theta>} \sigma^{\mathrm{T}}$ has elements $\left[\sigma \sqrt{<\delta+\Gamma \theta>} \sigma^{\mathrm{T}}\right]_{i j}$, $1 \leq i, j \leq n$. Then according to representation (2) elements of the covariance matrix of the state variables $Z$ are

$$
[\operatorname{Cov}(Z)]_{i j}=\int_{0}^{\infty}\left[\sigma \sqrt{<\delta+\Gamma \theta>} \sigma^{\mathrm{T}}\right]_{i j} e^{-\left(k_{i}+k_{j}\right) u} d u=\frac{\left[\sigma \sqrt{<\delta+\Gamma \theta>} \sigma^{\mathrm{T}}\right]_{i j}}{k_{i}+k_{j}},
$$

where $k_{i} \equiv(K)_{i i}>0$ is element of the main diagonal of a matrix $K$.

The zero coupon yield $y(\tau)$ according to representation (3) is linearly connected to state variables $Z$ by the relation $\tau y(\tau)=-A(\tau)+Z^{\mathrm{T}} B(\tau)$. Whence it follows that the variance of yield $y(\tau)$ it is calculated by the formula

$$
\operatorname{Var}[y(\tau)]=\left[B(\tau)^{\mathrm{T}} \operatorname{Cov}(Z) B(\tau)\right] / \tau^{2} .
$$

The equation (5) for vector function $B(\tau)$ for the examined case it is possible to write in the more convenient form

$$
b^{\prime}(\tau)=\mathbf{1}-\left[\widetilde{K}^{\mathrm{T}}+\widetilde{\Gamma}^{\mathrm{T}}<\lambda>\widetilde{\sigma}^{\mathrm{T}}\right] b(\tau)-\widetilde{\Gamma}^{\mathrm{T}}<b(\tau)^{\mathrm{T}} \widetilde{\sigma}>\widetilde{\sigma}^{\mathrm{T}} b(\tau) / 2, \quad b(0)=0 .
$$

where $b(\tau) \equiv \Phi^{-1} B(\tau)$ is independent on vector $\phi$. So if to pass to the state variables $\widetilde{Z}$, then it is possible to rewrite the formulae (10) and (11) as follows

$$
\begin{gathered}
y(\tau)=\left[-A(\tau)+Z^{\mathrm{T}} B(\tau)\right] / \tau=\left[-A(\tau)+\widetilde{Z}^{\mathrm{T}} b(\tau)\right] / \tau . \\
\operatorname{Var}[y(\tau)]=\left[b(\tau)^{\mathrm{T}} \Phi \operatorname{Cov}(Z) \Phi b(\tau)\right] / \tau^{2}=\left[b(\tau)^{\mathrm{T}} \operatorname{Cov}(\widetilde{Z}) b(\tau)\right] / \tau^{2} . \\
{[\operatorname{Cov}(\widetilde{Z})]_{i j}=\frac{\left[\widetilde{\sigma}<\delta+\Gamma \theta>\widetilde{\sigma}^{\mathrm{T}}\right]_{i j}}{k_{i}+k_{j}}, 1 \leq i, j \leq n .}
\end{gathered}
$$

By similar way it is possible to rewrite the formulae (6) and (7) for the forward rates and the forward rate spread.

From equality (6) it is possible to see that the forward rate can be presented in the form where the last term is stochastic and others are deterministic. Therefore, it is possibly to write that

$$
\begin{gathered}
\mathrm{E}[f(\tau \mid Z)]=r(\theta)-B(\tau)^{\mathrm{T}} \sigma<\delta+\Gamma \theta>\lambda-\left[B(\tau)^{\mathrm{T}} \sigma<\delta+\Gamma \theta>\sigma^{\mathrm{T}} B(\tau)\right] / 2, \\
\operatorname{Var}[f(\tau \mid Z)]=\Psi(\tau) \operatorname{Cov}(Z) \Psi(\tau)^{\mathrm{T}},
\end{gathered}
$$

where for brevity it is designed 


$$
\Psi(\tau) \equiv\left[\phi^{\mathrm{T}}-B(\tau)^{\mathrm{T}} K+B(\tau)^{\mathrm{T}} \sigma<\lambda>\Gamma-B(\tau)^{\mathrm{T}} \sigma<\sigma^{\mathrm{T}} B(\tau)>\Gamma / 2\right] .
$$

Derivative of forward rate curve for multifactor model

$$
\begin{aligned}
\frac{d f(\tau \mid Z)}{d \tau} & =-\left[B(\tau)^{\mathrm{T}}\right]^{\prime}\left[\sigma<\delta+\Gamma \theta>\lambda+\sigma<\delta+\Gamma \theta>\sigma^{\mathrm{T}} B(\tau)+\right. \\
+ & {\left.\left[K+\sigma<\lambda>\Gamma+\sigma<\sigma^{\mathrm{T}} B(\tau)>\Gamma\right](Z-\theta)\right], }
\end{aligned}
$$

its expectation and variance

$$
\begin{gathered}
E\left[\frac{d f(\tau \mid Z)}{d \tau}\right]=-\left[B(\tau)^{\mathrm{T}}\right]^{\prime}\left[\sigma<\delta+\Gamma \theta>\lambda+\sigma<\delta+\Gamma \theta>\sigma^{\mathrm{T}} B(\tau)\right], \\
\operatorname{Var}\left[\frac{d f(\tau \mid Z)}{d \tau}\right]=\left[B(\tau)^{\mathrm{T}}\right]^{\prime} \Theta \operatorname{Cov}(Z) \Theta^{\mathrm{T}}[B(\tau)]^{\prime},
\end{gathered}
$$

where for brevity it is designed $\Theta \equiv K+\sigma<\lambda>\Gamma+\sigma<\sigma^{\mathrm{T}} B(\tau)>\Gamma$.

From these formulae it is possible to see that the average slope of forward rate curve will be negative if functions $B(\tau)$ and their derivatives positive. Probability that for some term to maturity $\tau$ the slope of forward rate curve will be negative in this case can not be calculated because the probability distribution of state variable vector $Z$ is unknown. Note only that for the random variable of type $b^{\mathrm{T}} Z$ ( $b$ - some vector) Leippold $\& \mathrm{Wu}(1998)$ have obtained the characteristic function.

\section{The Brown - Schaefer Approximation}

At the analysis of the forward rate curves for long term yields Brown and Schaefer (2000) have offered approximation of the forward rate spread in this area in the form of simple dependence on the zero coupon yield volatility. This approximation is obtained if in the formula (7) to neglect the terms that depend linearly on $B(\tau)$. So the offered approximation is equivalent to that as the forward rate spread the following expression is used.

$$
\begin{gathered}
\Delta f(\tau, \omega) \equiv f\left(\tau_{2} \mid Z\right)-f\left(\tau_{1} \mid Z\right) \approx-\Delta B(\tau)^{\mathrm{T}} \sigma<\delta+\Gamma \theta>\sigma^{\mathrm{T}} \nabla B(\tau) / 2= \\
=-\left[b\left(\tau_{2}\right)-b\left(\tau_{1}\right)\right]^{\mathrm{T}} \widetilde{\sigma}<\delta+\Gamma \theta>\widetilde{\sigma}^{\mathrm{T}}\left[b\left(\tau_{2}\right)+b\left(\tau_{1}\right)\right] / 2 .
\end{gathered}
$$

Then the remainder $\varepsilon=-\Delta B(\tau)^{\mathrm{T}}[\sigma<\delta+\Gamma Z>\lambda+K(Z-\theta)]$ of the forward rate spread it is possible to consider as some error of approximation. It has the following properties. If the risk premium parameters to accept equal to zero $(\lambda=0)$ then the expectation of $\varepsilon$ will be equal to zero and its variance is equal

$$
\operatorname{Var}[\varepsilon]=\left[B\left(\tau_{2}\right)-B\left(\tau_{1}\right)\right]^{\mathrm{T}}\left[K \operatorname{Cov}(Z) K^{\mathrm{T}}\right]\left[B\left(\tau_{2}\right)-B\left(\tau_{1}\right)\right]
$$

If a probability distribution of $\varepsilon$ and its moments would be known it would be possible to construct confidential intervals and set the accuracy of approximation (13) at the set level of trust. Because the probability distribution is unknown it is possibly only to note that approximation will be better if a standard deviation of $\varepsilon$ (i.e. $d_{\varepsilon} \equiv \sqrt{\operatorname{Var}[\varepsilon]}$ ) will be smaller. In particular, if the order of smallness of $d_{\varepsilon}$ will be essentially higher than the order of the smallness of $\Delta f(\tau, \omega)$ then approximation can be considered as satisfactory.

Thus, accuracy of approximation will depend not only on the matrix $K$, the covariance properties of state variables, but also from functions of term structure $B(\tau)$. We shall consider this problem for two known multifactor models with a square root. 


\section{Three factor models}

Model BDFS (P. Balduzzi, S. Das, S. Foresi, R. Sundaram, 1996) relates to class $A_{1}(3)$, i.e. is three-dimensional model, in which as variables of a state are taken the short-term rate $r(t)$ (we shall consider its as variable $Z_{1}$ ), its local expectation $\theta(t)$ (a variable $Z_{2}$ ) and its local variance $v(t)$ (a variable $Z_{3}$ ). Then in our designations the BDFS model can be written as

$$
\left(\begin{array}{l}
d Z_{1} \\
d Z_{2} \\
d Z_{3}
\end{array}\right)=-\left(\begin{array}{ccc}
k_{1} & -k_{1} & 0 \\
0 & k_{2} & 0 \\
0 & 0 & k_{3}
\end{array}\right)\left(\begin{array}{c}
Z_{1}-\theta_{1} \\
Z_{2}-\theta_{2} \\
Z_{3}-\theta_{3}
\end{array}\right) d t+\left(\begin{array}{ccc}
1 & 0 & \sigma_{13} \\
0 & 1 & 0 \\
0 & 0 & \sigma_{33}
\end{array}\right)\left(\begin{array}{ccc}
\sqrt{Z_{3}} & 0 & 0 \\
0 & \sqrt{\zeta} & 0 \\
0 & 0 & \sqrt{Z_{3}}
\end{array}\right) d W,
$$

where $\theta_{1}, \theta_{2}, \theta_{3}$ - stationary expectation of corresponding variables of the state; $k_{1}, k_{2}, k_{3}, \sigma_{13}$, $\sigma_{33}, \zeta$ - positive constants; $W$-a vector of independent processes of the Brownian motions.

Thus, the specification of the equation (1) for this model is following

$$
K=\left(\begin{array}{ccc}
k_{1} & -k_{1} & 0 \\
0 & k_{2} & 0 \\
0 & 0 & k_{3}
\end{array}\right), \sigma=\left(\begin{array}{ccc}
1 & 0 & \sigma_{13} \\
0 & 1 & 0 \\
0 & 0 & \sigma_{33}
\end{array}\right), \delta=\left(\begin{array}{l}
0 \\
\zeta \\
0
\end{array}\right), \Gamma=\left(\begin{array}{lll}
0 & 0 & 1 \\
0 & 0 & 0 \\
0 & 0 & 1
\end{array}\right) .
$$

The Chen model (Chen, 1996) relates to class $A_{2}(3)$. The state variables at this model are the same processes $r(t), \theta(t)$ and $v(t)$, as in model BDFS, but the specification of the equation (1) for Chen model is another:

$$
K=\left(\begin{array}{ccc}
k_{1} & -k_{1} & 0 \\
0 & k_{2} & 0 \\
0 & 0 & k_{3}
\end{array}\right), \sigma=\left(\begin{array}{ccc}
1 & 0 & 0 \\
0 & \sigma_{22} & 0 \\
0 & 0 & \sigma_{33}
\end{array}\right), \delta=\left(\begin{array}{l}
0 \\
0 \\
0
\end{array}\right), \Gamma=\left(\begin{array}{lll}
0 & 0 & 1 \\
0 & 1 & 0 \\
0 & 0 & 1
\end{array}\right) .
$$

Because matrixes $K$ in both models are identical, the fundamental matrix of decisions for these models will be the same. Calculations give such result:

$$
U(t)=\left(\begin{array}{ccc}
e^{-k_{1} t} & \frac{k_{1}}{k_{1}-k_{2}}\left(e^{-k_{2} t}-e^{-k_{1} t}\right) & 0 \\
0 & e^{-k_{2} t} & 0 \\
0 & 0 & e^{-k_{3} t}
\end{array}\right) .
$$

Matrixes $\sigma<\delta+\Gamma \theta>\sigma \mathrm{T}$ that together with $U(t)$ determine the covariance matrix (2) for examined models have the following form.

For model BDFS:

$$
\sigma<\delta+\Gamma \theta>\sigma^{\mathrm{T}}=\left(\begin{array}{ccc}
\theta_{3}\left(1+\sigma_{13}^{2}\right) & 0 & \theta_{3} \sigma_{13} \sigma_{33} \\
0 & \zeta & 0 \\
\theta_{3} \sigma_{13} \sigma_{33} & 0 & \theta_{3} \sigma_{33}^{2}
\end{array}\right)
$$

For Chen model:

$$
\sigma<\delta+\Gamma \theta>\sigma^{\mathrm{T}}=\left(\begin{array}{ccc}
\theta_{3} & 0 & 0 \\
0 & \theta_{2} \sigma_{22}^{2} & 0 \\
0 & 0 & \theta_{3} \sigma_{33}^{2}
\end{array}\right) .
$$

Therefore the covariance matrixes (2) for these models have the following form. 
For model BDFS:

$$
\operatorname{Cov}[Z]=\left(\begin{array}{ccc}
\frac{\theta_{3}\left(1+\sigma_{13}^{2}\right)}{2 k_{1}}+\frac{\zeta k_{1}}{2 k_{2}\left(k_{1}+k_{2}\right)} & \frac{\zeta k_{1}}{2 k_{2}\left(k_{1}+k_{2}\right)} & \frac{\theta_{3} \sigma_{13} \sigma_{33}}{k_{1}+k_{2}} \\
\frac{\zeta k_{1}}{2 k_{2}\left(k_{1}+k_{2}\right)} & \frac{\zeta}{k_{2}} & 0 \\
\frac{\theta_{3} \sigma_{13} \sigma_{33}}{k_{1}+k_{2}} & 0 & \frac{\theta_{3} \sigma_{33}^{2}}{2 k_{3}}
\end{array}\right) .
$$

For Chen model:

$$
\operatorname{Cov}[Z]=\left(\begin{array}{ccc}
\frac{\theta_{3}}{2 k_{1}}+\frac{\theta_{2} \sigma_{22}^{2} k_{1}}{2 k_{2}\left(k_{1}+k_{2}\right)} & \frac{\theta_{2} \sigma_{22}^{2} k_{1}}{2 k_{2}\left(k_{1}+k_{2}\right)} & 0 \\
\frac{\theta_{2} \sigma_{22}^{2} k_{1}}{2 k_{2}\left(k_{1}+k_{2}\right)} & \frac{\theta_{2} \sigma_{22}^{2}}{2 k_{2}} & 0 \\
0 & 0 & \frac{\theta_{3} \sigma_{33}^{2}}{2 k_{3}}
\end{array}\right) .
$$

Thus, to calculate the variance (14) now it is necessary to determine only functions of term structure $B(\tau)$ from the equations (5). We shall consider this problem in the simplified variant, when a vector $\lambda$ of risk premium parameters is equal to zero. Then the equations (5) for examined models are reduced to the following.

For model BDFS:

$$
\begin{gathered}
B_{1}{ }^{\prime}(\tau)=1-k_{1} B_{1}(\tau), \quad B_{1}(0)=0 ; \\
B_{2}{ }^{\prime}(\tau)=1+k_{1} B_{1}(\tau)-k_{2} B_{2}(\tau), \quad B_{2}(0)=0 ; \\
B_{3}{ }^{\prime}(\tau)=1-k_{3} B_{3}(\tau)-B_{1}{ }^{2}(\tau) / 2-\left(\sigma_{13} B_{1}(\tau)+\sigma_{33} B_{3}(\tau)\right)^{2} / 2, \quad B_{3}(0)=0 .
\end{gathered}
$$

The solutions of first two equations are easy. Functions $B_{1}(\tau)$ and $B_{2}(\tau)$ are calculated in an explicit form:

$$
B_{1}(\tau)=\frac{1-e^{-k_{1} \tau}}{k_{1}}, B_{2}(\tau)=\frac{2}{k_{2}}-\frac{2 k_{1}-k_{2}}{k_{2}\left(k_{1}-k_{2}\right)} e^{-k_{2} \tau}+\frac{1}{k_{1}-k_{2}} e^{-k_{1} \tau} .
$$

However the equation (15) for $B_{3}(\tau)$ in an explicit form it is not possible to solve.

For Chen model:

$$
\begin{gathered}
B_{1}{ }^{\prime}(\tau)=1-k_{1} B_{1}(\tau), \quad B_{1}(0)=0 \\
B_{2}{ }^{\prime}(\tau)=1+k_{1} B_{1}(\tau)-k_{2} B_{2}(\tau)-\sigma_{22}^{2} B_{2}^{2}(\tau) / 2, \quad B_{2}(0)=0 \\
B_{3}{ }^{\prime}(\tau)=1-k_{3} B_{3}(\tau)-B_{1}^{2}(\tau) / 2-\sigma_{33}^{2} B_{3}^{2}(\tau) / 2, \quad B_{3}(0)=0 .
\end{gathered}
$$

In this case the function $B_{1}(\tau)$ is again determined simply, as well as in (16). But functions $B_{2}(\tau)$ and $B_{3}(\tau)$ cannot be determined in the explicit form.

The equations (15), (17) and (18) are the Riccati equations with variable factors and can be solved by the numerical methods only.

\section{Numerical analysis}

Now we use the formulae obtained above for calculation of some characteristics of models BDFS and Chen. 
In the paper Dai \& Singleton (2000) the empirical estimates of parameters for model BDFS and Chen model are reported (see Tables II and III). These data are following (designations are modified to our designations):

\begin{tabular}{cccccccc}
\hline Model & $k_{1}$ & $k_{2}$ & $k_{3}$ & $\theta_{1}$ & $\theta_{2}$ & $\theta_{3}$ & $\zeta$ \\
\hline BDFS & 2,05 & 0,0523 & 0,602 & 0,14 & 0,14 & 0,000156 & 0,000113 \\
Chen & 2,19 & 0,0757 & 1,24 & 0,0416 & 0,0416 & 0,000206 & 0 \\
\hline
\end{tabular}

$$
\sigma_{\text {BDFS }}=\left(\begin{array}{ccc}
1 & 0 & 3,533836 \\
0 & 1 & 0 \\
0 & 0 & 0,007197
\end{array}\right), \quad \sigma_{\text {Chen }}=\left(\begin{array}{ccc}
1 & 0 & 0 \\
0 & 0,050299 & 0 \\
0 & 0 & 0,019824
\end{array}\right) .
$$

Such data are sufficient for calculation of some characteristics. Further we give some examples that confirm this. At calculations it was accepted that risk premiums are absent $(\lambda=0)$.

Covariance matrix of processes $Z(t)$ of the state variables are calculated by formulae (2) in following form

$$
\begin{aligned}
\operatorname{Cov}[Z]_{\mathrm{BDFS}} & =\left(\begin{array}{ccc}
0,001567 & 0,001053 & 1,88 \times 10^{-6} \\
0,001053 & 0,002161 & 0 \\
1,88 \times 10^{-6} & 0 & 6,71 \times 10^{-9}
\end{array}\right), \\
\operatorname{Cov}[Z]_{\text {Chen }} & =\left(\begin{array}{ccc}
0,000719 & 0,000672 & 0 \\
0,000672 & 0,000695 & 0 \\
0 & 0 & 3,26 \times 10^{-7}
\end{array}\right) .
\end{aligned}
$$

The expectation of local variance of the yield process is determined by following formula $\mathrm{E}\left[\sigma_{y}(\tau) \sigma_{y}(\tau)^{\mathrm{T}}\right]=B(\tau)^{\mathrm{T}} \sigma<\delta+\Gamma \theta>\sigma B(\tau) / \tau^{2}$. The results of calculation of local variance of the yield process are presented on Figure 1 for model BDFS and Chen model.

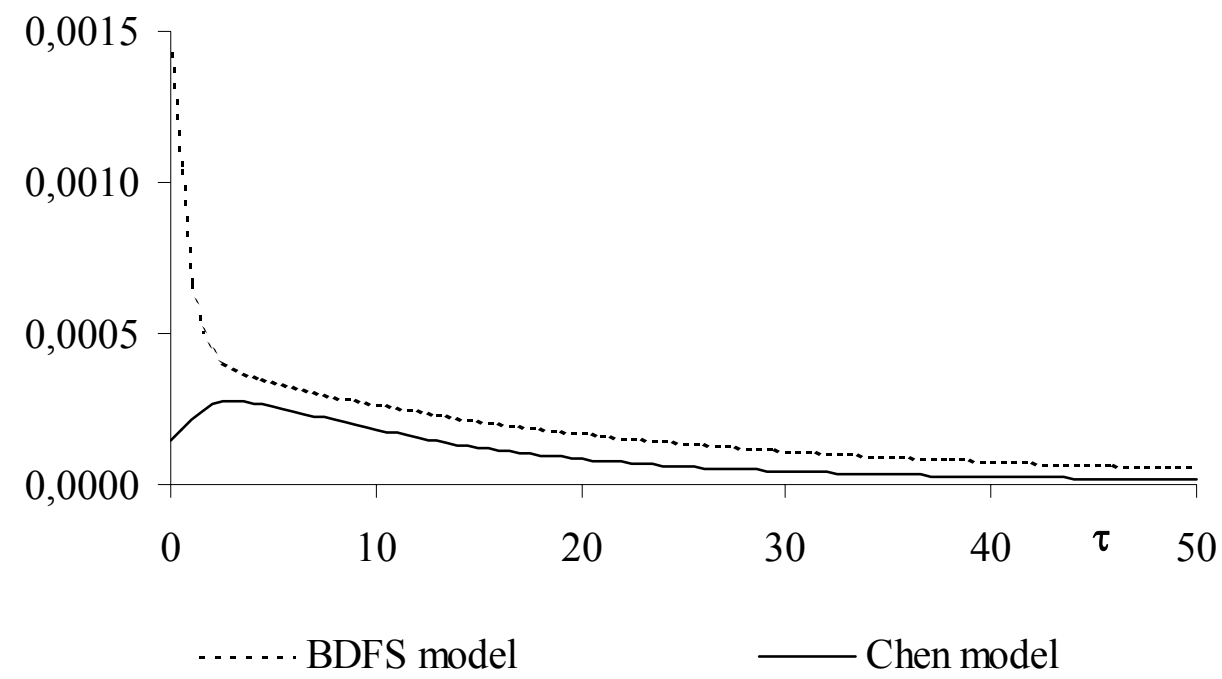

Figure 1. The expectation of local variance of the yield process $\mathrm{E}\left[\sigma_{y}(\tau) \sigma_{y}(\tau)^{\mathrm{T}}\right]$ as the function of term to maturity $\tau$ for BDFS model and Chen model. 
The variance of zero coupon yield $\operatorname{Var}[y(\tau)]$ is determined by expression (11). The results of calculation of $\operatorname{Var}[y(\tau)]$ are presented on Figure 2 for BDFS model and Chen model.

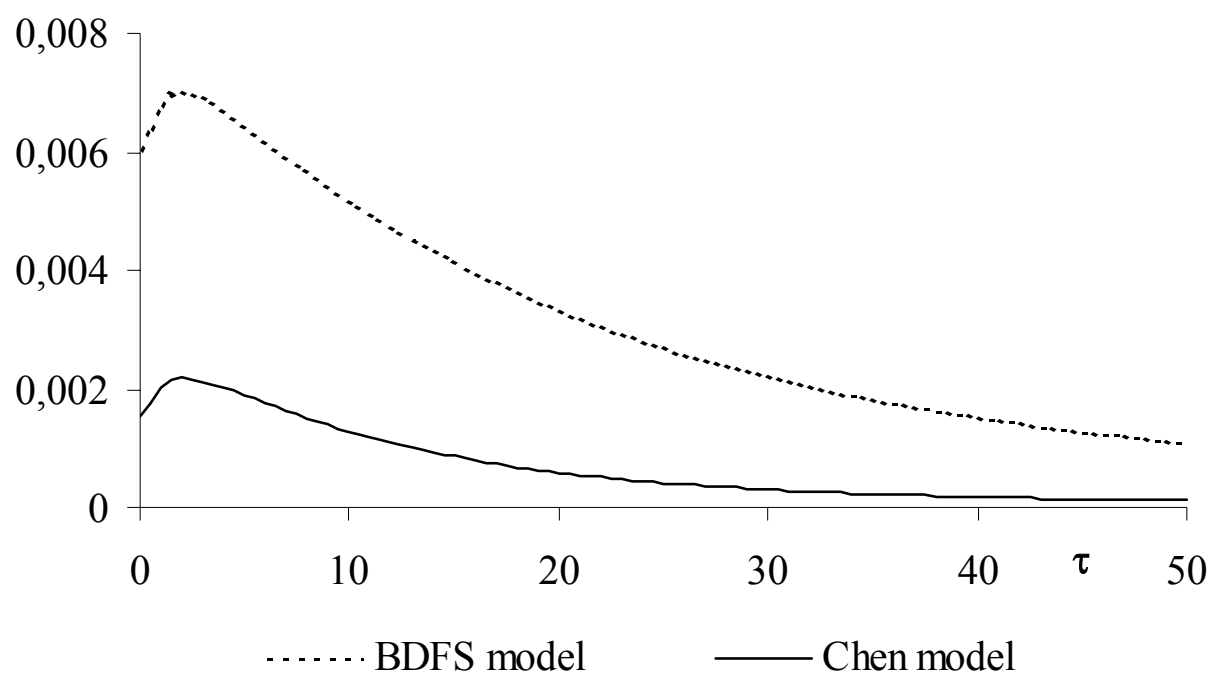

Figure 2. The variance of zero coupon yield $\operatorname{Var}[y(\tau)]$ as the function of term to maturity $\tau$ for BDFS model and Chen model.

By the similar way it is possible to calculate other characteristics of models.

\section{Conclusions}

In this paper the detailed analysis of multifactor models with stochastic volatility ("with square root") is resulted for the state variables that are generated by the equation (1). The problem connected to a negative slope of forward rate curves for the long term zero coupon yield rates was especially closely studied.

For multifactor models with stochastic volatility the following results are derived:

- representations of the forward rate curve through the volatility of the state variable process (6) and through the volatility of zero coupon yield process (8) are obtained;

- the expectations, variances and covariance matrixes for the forward rates and the yield process volatility are calculated;

- the expectation and the variance for the derivative of forward rate are found;

- the Brown - Schaefer approximation for the spread of forward rate is examined.

As examples two three-factor models (BDFS and Chen models) are examined. On the basis of the estimates of parameters of these models received by empirical way the numerical analysis including calculation of covariance matrixes of process of state variables, calculation of an expectation of the local variance of yield process (Figure 1) and calculation of the variance of zero coupon yield (Figure 2) have been fulfilled.

\section{References}

Balduzzi, P., Das, S., Foresi, S., and Sundaram, R. (1996) A Simple Approach to Three Factor Affine Term Structure Models. Journal of Fixed Income, 6, 43 - 53.

Brown, R. H. and Schaefer, S. M. (2000) Why Long Term Forward Interest Rates (Almost) Always Slope Downalds. Working paper. London Business School. 
Chen, L. (1996) Stochastic Mean and Stochastic Volatility - A Three Factor Model of the Term Structure of Interest Rate and Its Application to the Pricing of Interest Rate Derivatives. Blackwell Publisher.

Dai, Q. and Singleton, K. J. (2000) Specification Analysis of Affine Term Structure Models. Journal of Finance, 55(5), 1943-1978.

Duffie, D. and Kan, R. (1996) A Yield-Factor Model of Interest Rates, Mathematical Finance, 6, 379-406.

Hull, J. C. (1993) Options, Futures, and Other Derivative Securities. Prentice Hall, Englewood Cliffs.

Leippold, M. and Wu, L. (1998) The Potential Approach to Bond and Currency Pricing. Working paper. Swiss Institute of Banking and Finance.

Medvedev, G. A. (2003) Properties of Yield Curves and Forward Curves for Affine Term Structure Models. Proc. of the 13-th Intern. AFIR Symposium. Maastricht, 461-492.

Medvedev, G. A. (2004) Forward Interest Rates and Volatility of Zero Coupon Yield in Affine Models. Proc. of the 14-th Intern. AFIR Symposium. Boston. 